\section{First EurPhys}

The Register Commission for the professional designation European Physicist (abbreviated as Eur Phys) nominated following its first meeting on 3 November 1995 a total of 24 candidates for admission to the the European Register of Physicists. They were approved by the EPS Executive Committee so once registration fees are paid, each will receive a certificate and become eligible to use the designation Eur Phys. Among the nominations, six work in the UK and three in Germany.

EPS launched Eur Phys last year in response to a growing demand from physicists, especially those working in applied areas, who seek recognition of their pro-

The first certificates recognizing admission to the European Register of Physicists were handed over on 24 January at ceremonies in Switzerland and the UK. Left-hand photograph:M.J. Dutch, a plasma physicist working in Lausanne, receiving a Eur Phys certificate from M. Peter, on the left, who chairs the Register Commission; right-hand photograph: D.W. Thomas, a medical physicist who works in the Singleton Hospital in Wales, receiving a certificate from $H$. Schopper, on the left, the EPS President. fessional status. It is accorded on the basis of a minimum of three years' university education, two years' post-graduate experience and at least two years' additional education and/or post-graduation experience.

The European Monitoring Committee (EMC) accepts Eur Phys applications at any time, and makes recommendations to the Register Commission following regular meetings every 3-6 months. E.F. Slade, who is Director of Academic Affairs at Keele University, has accepted an invitation to chair the European Monitoring Committee, and Professor M. Peter, formerly Head of Geneva University's Physics Department, accepted a similar invitation to chair the Register Commission. A Code of Conduct has been approved by the
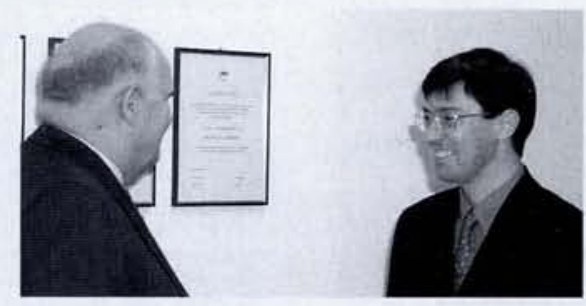

The international programme advisory committee responsible for the biennial International Conference on Accelerator and Large Experimental Physics Controls Systems (ICALEPCS), composed of representatives of the EPS Interdivisional Group for Experimental Physics Control Systems (EPCS) and other organizations active in the field, has decided that the 1997 conference will be held in Beijing with the Academia Sinica's Institute for High-Energy Physics (IHEP) as the organizer. The conference moves to Asia every six years (the last time was 1991 in Tsukuba, Japan), mainly because specialists from the region can make a valuable contribution to the conference, which with at least 300 participants is the largest international event of its type. This contribution stems from the region's increasing interest in large facilities. For instance, IHEP envisages re-engineering its $2 \mathrm{GeV}$ Beijing Electron-Positron Collider for charm and tau-lepton physics, synchrotron radiation and medium-energy nuclear physics. The institute also has plans to construct a high-lumi-nosity $3-6 \mathrm{GeV}$ tau-charm factory (a feasibility study is due finish by the end of 1996, with R\&D planned to start in 1997) as well as a synchrotron radiation facility in a joint project with the Institute of Nuclear Physics, Shanghai. Korea, which has the Pohang Light Source (PLS) consisting of a 2 $\mathrm{GeV}$ electron linear accelerator and a $2 \mathrm{GeV}$ low-emittance storage ring, intends to build a superconducting tokamak comparable to Princeton's TPX device. Finally, in addition to a synchrotron source in Taiwan, India's Variable Energy Cyclotron Centre, and the Institute for Nuclear Physics in Novosibirsk in the eastern part of the CIS, there are several major experimental facilities (accelerators, fusion devices, lasers, etc.) in Japan.

A. Daneels, an EPCS representative on the ICALEPCS advisory committee, reports that IHEP offered to be the 1997 host immediately after the 1993 event in Berlin.

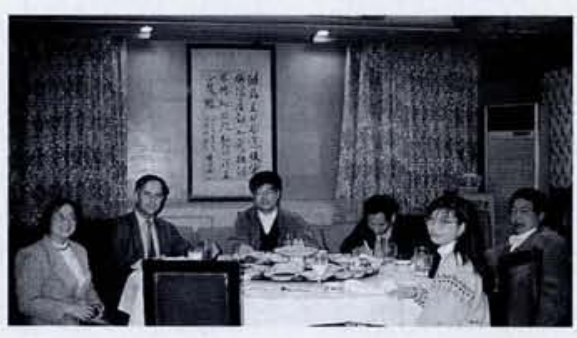

A. Daneels, second from left, met with IHEP and Academia Sinica officals in October 1995.
Executive Committee, which also agreed that appeals against refusal to admit an applicant to the Register be referred to the President of EPS. The Commission has recommended that a reserve fund be created to help cover the fees of candidates, notably those based in economically weak regions of Europe.

As the Register grows, the aim is to continue to ensure a uniform high standard for entry by candidates that have worked and been educated throughout Europe. For with the successful launch of Eur Phys it is clear that the designation meets the expectations of physicists needing additional professional recognition. Contacts: E.W.A. Lingeman (ed@nikhef.nl; tel: +31-20-592 21 17; fax: +31-20-592 62 65); EurophysNet at http://epswww.epfl.ch/

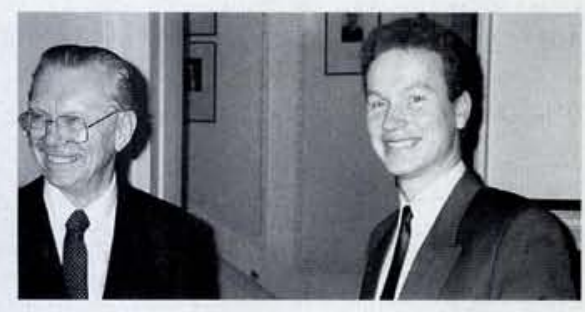

However, as there have been some reservations about holding an international technologically oriented event such as ICALEPCS in Asia, and more specifically in China, the advisory committee made a second call for proposals. This proved successful and besides the IHEP which reiterated its offer, additional proposals came from laboratories in India, Korea and Russia of whom all but one have been EPCS members for several years. A 1993 offer from Taiwan was not repeated.

The IHEP was selected, not only because it is one the region's more active EPCS members but also because it offered a purpose-built conference centre of international standard and with good communications facilities (an important aspect for industrial exhibitors). Moreover, Beijing can provide good-value accommodation comparable to that found elsewhere in the region and a wealth of cultural landmarks. The IHEP itself offered grants for 20-30 participants from less-developed countries, a stimulating technical environment, general support from the General Office of the Academia Sinica, and help for infrastructure, logistics and the cultural programme from China's Office of Foreign Affairs. Finally, the IHEP boasts a long experience in international conferences, including the 1995 International Symposium on Lepton-Photon Interactions that attracted some 1000 participants. 